\title{
Maternal and fetal outcomes of pregnancy with Fontan circulation: A multicentric observational study
}

\author{
Marielle Gouton a,b , Jacky Nizard ${ }^{\mathrm{c}}$, Mehul Patel ${ }^{\mathrm{d}}$, François Sassolas ${ }^{\mathrm{e}}$, Maria Jimenez ${ }^{\mathrm{f}}$, Jelena Radojevic ${ }^{g}$, \\ Amel Mathiron ${ }^{\mathrm{h}}$, Pascal Amedro ${ }^{\mathrm{i}}$, Elise Barre ${ }^{\mathrm{j}}$, Fabien Labombarda ${ }^{\mathrm{k}}$, Guy Vaksmann ${ }^{\mathrm{l}}$, Alain Chantepie ${ }^{\mathrm{m}}$, \\ Laurianne Le Gloan ${ }^{\mathrm{n}}$, Magalie Ladouceur ${ }^{\mathrm{a}, \mathrm{o}, *}$
}

a Centre de Référence des Malformations Cardiaques Congénitales Complexes, M3C Paris, France

${ }^{\mathrm{b}}$ Adult Congenital Heart Disease Unit, Centre Chirurgical Marie-Lannelongue, Le Plessis-Robinson, France

c Service de Gynécologie Obstétrique, Groupe Hospitalier Pitié-Salpêtrière, APHP, Sorbonne Universités, UPMC Univ Paris 06, CNRS UMR 7222, INSERM U1150-, Paris, France

d Baylor College of Medicine, Houston, TX, USA

e Cardiologie Pédiatrique et Congénitale Adulte, Hôpital Cardiovasculaire Louis Pradel, Lyon, France

${ }^{\mathrm{f}}$ Pediatric Department, Clinique Saint Augustin, Bordeaux, France

g Cardiologie Congénitale, Strasbourg, France

h Pediatric Cardiology Department, Hôpital Nord, Amiens, France

${ }^{i}$ Pediatric Department 1, Hôpital Arnaud de Villeneuve, Montpellier, France

${ }^{j}$ Pediatric Department 1, Centre Hospitalo-Universitaire de Rouen, Rouen, France

${ }^{\mathrm{k}}$ Department of Cardiology, CHU de Caen, Avenue Côte-de-Nacre, Caen, France

${ }^{1}$ Maladies Cardiovasculaires Infantiles et Congénitales, Lille, France

${ }^{\mathrm{m}}$ Pediatric Department, Hôpital Clocheville, Tours, France

n Cardiologie Congénitale Adulte et Pédiatrique, Hôpital Mère-enfant-maternité, Nantes, France

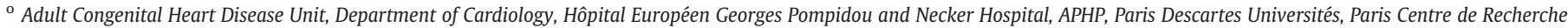

Cradiovasculaire, INSERM U970, Paris, France

\section{A B S T R A C T}

Background: Despite serious long-term sequel, women with Fontan palliation have reached childbearing age. However there is paucity of data on the pregnancy outcomes and management of this condition. We aimed to determine the maternal and fetal outcomes of pregnancy in women with Fontan palliation.

Methods: This multicentric, retrospective study included women with Fontan circulation followed in 13 French specialized centers from January 2000 to June 2014. All pregnancies were reviewed, including miscarriages, abor-tions, premature and term births. We reviewed maternal and fetal outcomes.

Results: Thirty-seven patients had 59 pregnancies. Mean age was $27 \pm 5$ years at first pregnancy. There were 16 miscarriages (27\%) and 36 live births with 1 twin pregnancy. Cardiac events occurred in $6(10 \%)$ pregnancies, with no maternal death. The most common cardiac complication was atrial arrhythmia, which occurred in 3 pa-tients. Hematological complications including thromboembolic/hemorrhagic events $(n=3 / 7)$ occurred in 5 women antepartum ( $n=2 / 3$ ), and 4 women postpartum $(n=1 / 4)$. Two of the 3 thromboembolic events oc-curred in patients without anticoagulation. There was a high incidence of prematurity $(\mathrm{n}=25 / 36,69 \%)$. Anticoagulation was associated with adverse neonatal outcome ( $\mathrm{OR}=10.0,95 \% \mathrm{CI}[1.5-91.4], \mathrm{p}<0.01)$. After a median followup of 24 months, there was no significant worsening of clinical status and thromboembolic disease noted.

Conclusions: Pre-selected women can successfully complete pregnancy with Fontan circulation. There is an increase in cardiac and neonatal morbidity during pregnancy. Because thromboembolism could have a severe consequence on Fontan circulation, anticoagulation should be indicated during pregnancy and postpartum period.

Keywords:

Pregnancy

Fontan circulation

Congenital heart disease

Anticoagulation

\footnotetext{
* Corresponding author at: Adult Congenital Heart Disease Unit, Department of Cardiology, Hôpital Européen Georges Pompidou, 20 Rue Leblanc, 75015 Paris.

E-mail address: magalie.ladouceur@egp.aphp.fr (M. Ladouceur).
}

\section{Introduction}

Since 1971, Fontan procedure has been adopted worldwide for the management of patients with complex congenital heart disease considered unsuitable for a biventricular repair. Surgical evolution of this 
technique [1-3] has significantly improved survival for patients with functional univentricular heart. However, intrinsic to the construction of the Fontan circulation, secondary complications due to extensive surgical distortion of cardiac anatomy and physiology are inevitable. The wide spectrum of complications includes arrhythmia, ventricular dysfunction, thromboembolic events, hepatic dysfunction, and protein losing enteropathy. Nevertheless, long-term survival is good $[4,5](90 \%$ at 10 years, $83 \%$ at 20 years, and $70 \%$ to $76 \%$ at 25 years) and an increasing number of women reaching childbearing age following surgery express desire to have children. Hemodynamic changes in pregnancy are pronounced for the abnormal heart (cardiac output and stroke volume double, myocardial oxygen consumption increases by $20 \%$, heart rate increases by $15-20 \%)$. Paucity of data on the maternal and fetal risks, as well as short and long-term outcomes, leaves preconception counseling with many unanswered questions, most importantly on the type and duration of anticoagulation to prevent thromboembolic accidents [6]. Thromboembolic complications during pregnancy are expected to increase, as Fontan state and pregnancies are both pro-thrombotic milieu $[7,8]$. However, no study has shown that anticoagulation reduces the risk in this context. Moreover, the late consequences of pregnancy on the Fontan heart need to be analyzed given the limited data on long-term survival [4,5]. Published literature includes anecdotal case reports and small series [9-11], which are insufficient to evaluate and identify predictors of maternal risk during pregnancy, to provide recommendations, and subsequently guide providers for counseling.

The aim of this study was to determine the fetal and maternal outcomes of pregnancy in women with Fontan palliation and impact of pregnancy on heart disease at last follow-up in a large series of patients.

\section{Methods}

\subsection{Population}

All pregnant women with Fontan palliation registered in one of the 13 centers of the French M3C network (Centres de Référence et de Compétence des Malformations Cardiaques Congénitales Complexes, M3C) during the time period from January 2000 to June 2014 were included in the study. We did not include ongoing pregnancies by the end of the study period. For each pregnancy, the cardiac and obstetric records were reviewed.

Baseline data included initial heart anatomy, prior surgical procedures, cardiac complications before pregnancy, age at first pregnancy, infertility (more than two years of pregnancy attempts, investigated and documented by a gynecologist), miscarriages (spontaneous fetal loss before 20 week gestation (WG)), first trimester elective abortions, and terminations of pregnancy.

Detailed information concerning each completed (>20 WG) pregnancy was recorded along with antepartum, peripartum and postpartum data when applicable. Anticoagulation treatment, mode of delivery, parity status, and New York Heart Association (NYHA) functional class were also recorded.

Documented complications were divided into maternal cardiac, obstetrical and neonatal complications. Hematological complications were described during the antepartum and postpartum periods. Cardiac complications were documented as arrhythmia or heart failure requiring treatment, systemic thromboembolic complication, new cyanosis (oxygen saturation $<92 \%$ at rest) and protein-losing enteropathy. Hematological complications included any documented episode of venous thromboembolic disease, including pulmonary embolism, and hemorrhagic events as abruptio placentae, and postpartum hemorrhage (documented $\geq 500 \mathrm{ml}$ for vaginal delivery, $\geq 1000 \mathrm{ml}$ for CS within the first $24 \mathrm{~h}$ postpartum). Obstetrical complications included pregnancyinduced hypertension (occurring after $>20 \mathrm{WG}, \geq 140 \mathrm{~mm}$ Hg systolic or $90 \mathrm{~mm} \mathrm{Hg}$ diastolic without proteinuria); pre-eclampsia (pregnancyinduced hypertension with proteinuria $\geq 0.3 \mathrm{~g} / 24 \mathrm{~h}$ urine sample); eclampsia; hemolysis, raised liver enzymes (transaminitis), low platelet (HELLP) syndrome; gestational diabetes mellitus; premature rupture of membranes before term (membrane rupture before the onset of uterine contractions before $37 \mathrm{WG}$ ); premature labor (spontaneous onset of labor before 37 WG); and cesarean section (CS). Fetal and neonatal complications included premature delivery (delivery before $37 \mathrm{WG}$ ); small for gestational age birth weight $(<10$ th percentile); stillbirth ( $>20$ WG); neonatal death (death within the first month after birth) and recurrence of congenital heart disease diagnosed by antenatal echocardiography and confirmed at birth by a pediatric cardiologist, or diagnosed by postnatal echocardiography.

Maternal cardiac complications were also recorded at last follow-up.

\subsection{Statistical analysis}

Descriptive statistics for nominal data were expressed as absolute numbers and percentages. Means (SD) were calculated for continuous variables. Medians and quartiles were computed for continuous variables with non-normal distribution. Association between basal characteristics of women and cardiovascular, neonatal, and obstetric events was assessed using the $\chi^{2}$ test or Fisher test for nominal data, and Wilcoxon test for continuous data. Two-tailed probability values $\leq .05$ were considered statistically significant. Statistical testing was performed with the use of MedCalc Statistical Software version 12.7.7 (MedCalc Software bvba, Ostend, Belgium; 2013).

\section{Results}

\subsection{Baseline characteristics}

During the study period, 37 women had a total 59 pregnancies. Mean age at first pregnancy was $27 \pm 5$ years ( 19 to 41 years). Thirteen women had more than one pregnancy (up to 5 pregnancies). Underlying congenital heart lesions and maternal baseline characteristics are summarized in Tables 1 and 2.

Half of the population had tricuspid atresia, and half of palliations were intracardiac total cavo-pulmonary connection (TCPC). Mean age at Fontan palliation was $13 \pm 7$ years. Seven Fontan or Kreutzer conversions were performed before pregnancies; one woman underwent Fontan conversion between her second pregnancy.

Pregestational complete clinical information on cardiac events was available for all women. Seven women had atrial arrhythmia before being pregnant: 4 had atrial flutter and 3 atrial tachycardia. One woman with tricuspid atresia received an internal cardioverter defibrillator before pregnancy because of systemic ventricle dysfunction and sustained ventricular tachycardia on 24-hour Holter ECG monitoring.

\section{Table 1}

Native congenital heart disease and single ventricle palliation characteristics. DILV: double inlet left ventricle, DORV: double outlet right ventricle, SV: single ventricle, TCPC: total cavo-pulmonary connection.

\begin{tabular}{ll}
\hline Women & $\mathrm{n}=37(\%)$ \\
\hline CHD & \\
Tricuspid atresia & $19(51 \%)$ \\
DILV & $7(19 \%)$ \\
DORV & $3(8 \%)$ \\
SV & $7(19 \%)$ \\
VSD & $1(3 \%)$ \\
Type of palliation & \\
Extracardiac TCPC & $11(30 \%)$ \\
Intracardiac TCPC & $20(54 \%)$ \\
Fontan & $4(11 \%)$ \\
Kreutzer & $2(5 \%)$ \\
Mean age ( \pm SD) at Fontan palliation (y) & $16 \pm 7$ \\
Fontan or Kreutzer conversion before pregnancy & 7
\end{tabular}


Table 2

Maternal baseline characteristics. TCPC: total cavo-pulmonary connection.

\begin{tabular}{ll}
\hline Demographics & \\
\hline Age at first pregnancy (mean \pm SD) in years & $27 \pm 5$ \\
Functional state & $21(57 \%)$ \\
NYHA1 & $16(43 \%)$ \\
NYHA2 & 0 \\
NYHA 3 and 4 & 1 \\
Hypoxemia (saturation <92\%) & \\
Past medical history & 7 \\
Atrial arrhythmia & 1 \\
Permanent pace maker & 1 \\
Internal cardioverter defibrillator & 3 \\
Heart failure & 7 \\
Thromboembolism & \\
Hemodynamics & $33(89 \%)$ \\
Normal systemic ventricular function & $4(11 \%)$ \\
Mild systemic ventricle dysfunction & 0 \\
Severe systemic ventricle dysfunction & $34 / 3 / 0 / 0$ \\
Atrio-ventricular valve regurgitation grade I/II/III/IV & 0 \\
TCPC abnormal flow &
\end{tabular}

Three women perceived palpitations without documented arrhythmia. Thromboembolism occurred in 8 patients before pregnancy: 2 were pulmonary embolisms, of which one occurred while under combined oral contraceptive treatment, two were deep venous thrombosis, two were intracardiac thrombi diagnosed before Fontan conversion, and two were strokes, with one occurring during atrial arrhythmia and one before Fontan palliation. Transient congestive signs were diagnosed in 2 women with normal systemic ventricle function, one just after TCPC and one after fenestration closure. All patients with medical history of atrial arrhythmia or thromboembolism were treated by therapeutic oral anticoagulation before pregnancy (INR between 2 and 3).

One patient had cyanosis (basal saturation before pregnancy $=89 \%$ ) because of veno-venous fistula.

\subsection{Infertility}

Six (21\%) of 29 for whom data were available experienced infertility and required ovulation stimulation $(4 / 6)$ or an in vitro fertilization $(1 / 6)$. The sixth one was a patient who was diagnosed with a spontaneously monochorial biamniotic twin pregnancy, at the prestimulation visit.

\subsection{Cardiovascular complications during pregnancy and postpartum}

Qualifying cardiac events (Table 3) occurred in 6 of the 59 pregnancies $(10 \%)$ during pregnancy and 3 women during the postpartum period.

During pregnancy, the most common adverse cardiovascular events were atrial arrhythmia.

Arrhythmias were documented in 3 patients: 2 were paroxysmal atrial arrhythmias and one was persistent atrial fibrillation. Persistent

Table 3

Maternal complications during pregnancy. PLE: protein losing enteropathy.

\begin{tabular}{lll}
\hline Type & $\mathrm{N}$ & Term (trimester) \\
\hline Supra ventricular tachycardia & 3 & 1st and 2nd \\
Systemic thrombo-embolism & 2 & 1st and 3rd \\
Heart failure & 4 & 3rd and postpartum \\
New hypoxemia (sat $<92 \%)$ & 1 & 3rd \\
Others & & \\
$\quad$ Renal failure & 1 & 2nd \\
Anemia (requiring transfusion) & 1 & 3rd \\
PLE & 0 & - \\
Maternal death & 0 & \\
\hline
\end{tabular}

atrial fibrillation occurred at 25 WG in a woman with a Fontan palliation, and led to heart failure at 32 weeks. Chemical cardioversion with amiodarone was successful. The 2 paroxysmal atrial arrhythmias ( 1 flutter and 1 atrial fibrillation) were treated by beta-blockers (sotalol and propranolol). All patients with arrhythmia received therapeutic anticoagulation. Past medical history of arrhythmia was significantly predictive of atrial arrhythmia during pregnancy $(\mathrm{OR}=18.3 ; 95 \% \mathrm{CI}$ [0.8-1212.7]; $\mathrm{p}=0.03$ ), while no association was found with the type of palliation. No other predictor of atrial arrhythmia during pregnancy was found. No patient had ventricular arrhythmia.

Two patients experienced systemic thromboembolism complications during the antepartum period. None of them had history of thromboembolism before pregnancy. A woman with tricuspid atresia palliated by intracardiac TCPC had a transient ischemic attack in the first trimester. No atrial arrhythmia was documented, and she was initially treated by antiplatelet drugs alone. Another thromboembolic event occurred in a woman with a double outlet right ventricle palliated by extra cardiac TCPC in which an intra-cardiac thrombus was diagnosed by echocardiography at 18 WG. Before diagnosis, she was treated by preventive anticoagulation with enoxaparin (4000 anti-Xa IU/24 h) started at 13 WG. She had no other thrombo-embolic complication during the pregnancy and until her last follow-up.

Heart failure occurred in 3 women during the postpartum period, one occurred 15 days after delivery in a 31 year-old woman with tricuspid atresia palliated by an intracardiac TCPC. Echocardiography disclosed a moderate left ventricular dysfunction. Another one occurred in the previously described patient with Fontan who experienced atrial fibrillation and heart failure during pregnancy, and one after blood transfusion. No arrhythmia or systemic thromboembolism occurred during this period.

There was no maternal mortality in our population during pregnancy.

\subsection{Obstetrical and neonatal events}

Among the 59 pregnancies, 4 ended in elective abortion and 16 in miscarriage (13 during the first trimester, 3 during the second), at a mean gestational age of $9.6 \pm 4.7$ WG. Types of palliation, maternal age, NYHA functional class, past cardiac event and treatment during pregnancy, including anticoagulation or antiarrhythmic drugs, were not predictive of miscarriage.

All 59 pregnancies were singletons except for 1 twin pregnancy (delivered by CS at 32 WG). Thirty-nine (66\%) pregnancies were completed ( $\geq 20 \mathrm{WG}$ ), with a mean gestational age at delivery of $34 \pm 4 \mathrm{WG}$ (minimum $=25 \mathrm{WG}$, maximum $=38 \mathrm{WG}$ ). One late intrauterine fetal death occurred at 35 WG in a 22 year-old woman with a tricuspid atresia palliated by Fontan procedure who accidentally received an angiotensin converting enzyme inhibitor during pregnancy from an outside physician. The fetus had single ventricle with severe ventricular dysfunction leading to intrauterine death. One stillbirth occurred at 21 WG in relation with an abruptio placentae and a premature rupture of membranes before term.

The only neonatal death occurred in a patient who had a history of 4 prior miscarriages. During pregnancy monitoring, an abnormal uterine Doppler flow and a small gestational age fetus were diagnosed at 18 WG. The pregnancy was complicated by premature rupture of membranes at 17 WG, and by a chorioamnionitis at 25 weeks needing extraction by CS. The neonate weight was $510 \mathrm{~g}$ ( $<10$ th percentile); he died 15 days after delivery from complications of extreme prematurity.

Among the 38 completed pregnancies, 16 (42\%) were delivered by CS (including the twin pregnancy), and 22 (58\%) had successful vaginal delivery. General anesthesia was performed for 8 of the 16 deliveries by $\mathrm{CS}$. The other patients who had a cesarean delivery had either an epidural $(n=3)$ or a spinal anesthesia $(n=5)$. All vaginal births were done under epidural analgesia. 
Table 4

Obstetrical complications and neonatal outcomes. CHD: congenital heart disease, WG: week gestation; LSVC: left superior vena cava, SV: single ventricle.

\begin{tabular}{ll}
\hline Obstetrical complications & $\mathrm{n}$ \\
\hline Miscarriage & $16(27 \%)$ \\
Chorioamnionitis & 1 \\
Transaminitis $(\times 3)$ & 1 \\
Preeclampsia & 1 \\
Premature rupture of membranes & 7 \\
Preterm labor & 4 \\
Postpartum hemorrhage & 3 \\
& \\
Fetal/neonatal complications & $\mathrm{N}$ \\
\hline Live births & $38(1 \mathrm{twin}$ pregnancy) \\
Birth weight (g), mean \pm SD & $1968 \pm 74$ \\
Fetal/neonatal complications & \\
Intrauterine fetal death & 1 \\
Stillbirth & 1 \\
Neonatal death & 1 \\
Fetal distress & 4 \\
SGA (<10th percentile) & 4 \\
Prematurity (birth $<37$ WG) & 25 (69\%) \\
33-36 + 6 WG & 15 \\
$28-32+6$ WG & 7 \\
$22-27+6$ WG & 3 \\
Recurrence of CHD & $2(1 \mathrm{LSVC}, 1 \mathrm{SV})$ \\
\hline & \\
&
\end{tabular}

The mean birth weight was $1968 \pm 74 \mathrm{~g}$ at a mean gestational age of $34 \mathrm{WG}$, which corresponds to the 10th percentile.

Obstetric and neonatal events are summarized in Table 4 . The most common neonatal complication was prematurity ( $n=25 / 36,69 \%$ of neonates). Two neonates (5.6\%) were diagnosed with cardiac anomalies: one with single ventricle and one with left superior vena cava. All cases were identified in utero by fetal cardiac ultrasonography and the left superior vena cava was confirmed by echocardiography at birth. No additional congenital malformation was diagnosed later in the follow-up.

\subsection{Hematological complications}

\subsubsection{Anticoagulation regimens (Table 5)}

We clustered patients in 3 groups according to anticoagulation regimen: 1) patients without any anticoagulation therapy during pregnancy $(\mathrm{n}=13)$. In this group, 9 women were treated by aspirin alone (100-150 g a day). 2) Women under prophylactic anticoagulation with LMWH (enoxaparin 4000 anti-Xa IU/24 h, or 4000 anti-Xa IU/ $12 \mathrm{~h}$, or fraxiparine 2850 anti-Xa IU/24 h), or subcutaneous injection of UFH (calcium heparin $5000 \mathrm{IU} / 12 \mathrm{~h}$ ). Doses of LMWH were chosen by the practitioner according the estimation of thromboembolic risk (abnormal Fontan circulation flow, systemic ventricular dysfunction, echocardiographic contrast in the heart or in the vena cava). There was no anticoagulation monitoring in women under this anticoagulation therapy, but platelet levels were controlled monthly. 3) Women under curative anticoagulation with AVK or LMWH (enoxaparin $100 \mathrm{IU}$ antiXa/kg/12 h or tinzaparin $175 \mathrm{IU}$ anti-Xa IU/kg/24 h) or UFH (calcium heparin $500 \mathrm{IU} / \mathrm{kg} / 24 \mathrm{~h}$ ). A monthly anticoagulation monitoring was performed. Platelet levels were also controlled monthly in women under heparin. Doses were adjusted according to weight and for a PTT $\geq 2$ times the control, 4 to $6 \mathrm{~h}$ after starting in patients under UFH, a INR between 2 and 3 in women treated by AVK, and anti-Xa levels between $0.5-1 \mathrm{U} / \mathrm{ml}$, determined 4 to $6 \mathrm{~h}$ after application of $\mathrm{LMWH}$.

\subsubsection{Antepartum period}

No venous thromboembolic disease was diagnosed during the antepartum period. Abruptio placentae was diagnosed in 3 pregnancies treated respectively by antiplatelet therapy, UFH prophylactic anticoagulation and LMWH therapeutic anticoagulation. Anticoagulation was stopped 24 to $48 \mathrm{~h}$ before programmed delivery, and restarted at 24-48 h.

\subsubsection{Postpartum period}

A 24 year-old woman with tricuspid atresia palliated by extracardiac TCPC, and with known veno-venous fistulae, experienced Fontan failure secondary to pulmonary embolism during the postpartum. During pregnancy, she received therapeutic anticoagulation with tinzaparin, with a monthly antiXa monitoring, until 27 WG when she spontaneously interrupted the treatment. She underwent an emergency cesarean section at 31 WG for premature rupture of membranes and progression of congestive signs. Twenty-four hours after delivery, she experienced a sudden onset of dyspnea and loss of consciousness requiring resuscitation with respiratory support. Sub-massive pulmonary embolism was confirmed by computed tomography (CT). The patient was treated by heparin and coumadin. Laboratory investigations showed a decrease of serum albumin level ( $18 \mathrm{~g} / \mathrm{l})$, and Doppler observed an impairment of Glenn flow. TCPC function was restored and albumin levels normalized one month after delivery, however her functional status stepped up from NYHA I before pregnancy to NYHA 2 after this accident.

Three postpartum hemorrhages occurred, one in a woman treated by antiplatelet therapy, and in 2 women treated by therapeutic anticoagulation stopped at least $12 \mathrm{~h}$ before delivery. One abdominal wall hematoma complicated a CS, in a woman treated by therapeutic anticoagulation. There were no late hematological complications.

Table 5

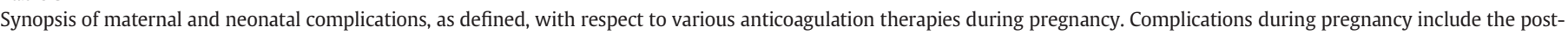

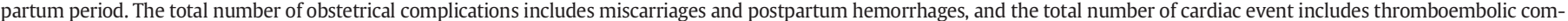
plications. LMWH: low molecular weight heparin, UFH: unfractionated heparin.

\begin{tabular}{|c|c|c|c|c|c|c|c|}
\hline \multirow[t]{2}{*}{ Type of anticoagulation } & \multirow[t]{2}{*}{$\mathrm{n}$} & \multicolumn{3}{|c|}{ Maternal complications during pregnancy } & \multirow{2}{*}{$\begin{array}{l}\text { Late maternal } \\
\text { complications }\end{array}$} & \multicolumn{2}{|c|}{ Neonatal outcomes } \\
\hline & & $\begin{array}{l}\text { Cardiovascular } \\
\text { complications }\end{array}$ & $\begin{array}{l}\text { Obstetrical } \\
\text { (miscarriages) }\end{array}$ & Hemorrhagic/thrombotic & & $\begin{array}{l}\text { Live } \\
\text { births }\end{array}$ & $\begin{array}{l}\text { Neonatal } \\
\text { complications }\end{array}$ \\
\hline \multicolumn{8}{|l|}{ No anticoagulation } \\
\hline No anticoagulation & 4 & 2 & $1(0)$ & $0 / 1$ & 2 & 4 & 1 \\
\hline Antiplatelet therapy & 11 & 1 & $4(3)$ & $2 / 1$ & 1 & 8 & 3 \\
\hline \multicolumn{8}{|c|}{ Prophylactic anticoagulation } \\
\hline LMWH & 11 & 2 & $2(1)$ & $0 / 1$ & 2 & 8 & 7 \\
\hline UFH & 1 & 0 & $1(0)$ & $1 / 0$ & 1 & 0 & 1 \\
\hline \multicolumn{8}{|c|}{ Therapeutic anticoagulation } \\
\hline LMWH & 16 & 3 & $7(3)$ & $3 / 0$ & 0 & 13 & 10 \\
\hline UFH & 1 & 1 & $1(0)$ & $0 / 0$ & 0 & 1 & 1 \\
\hline AVK & 10 & 0 & $6(4)$ & $0 / 0$ & 1 & 4 & 3 \\
\hline Unknown treatment & 5 & 0 & $5(5)$ & $0 / 0$ & 0 & 0 & 0 \\
\hline Total & 59 & 9 & $27(16)$ & $6 / 3$ & 7 & 38 & 26 \\
\hline
\end{tabular}




\subsection{Anticoagulation during pregnancy}

Table 5 summarizes the outcomes of fetal and maternal complications, according to the type of anticoagulation during pregnancy. In total 25/54 pregnancies (46\%) were treated by therapeutic anticoagulation and 12 (22\%) by prophylactic anticoagulation. Among the 3 thromboembolic events, one occurred under preventive anticoagulation with LMWH (enoxaparin 4000 anti-Xa $\mathrm{IU} / 24 \mathrm{~h}$ ) and 2 in patients without anticoagulation. Spontaneous abortion occurred in $26 \%$ in women with curative anticoagulation, $5 \%$ in women with preventive anticoagulation and $20 \%$ in women without anticoagulation. These miscarriages were more common in women treated by AVK ( $40 \%$ vs. $27 \%$ in the group treated by antiplatelet therapy and $15 \%$ in the group treated by LMWH). Anticoagulation treatment was unknown in 5 cases of miscarriage. We observed no fetal malformation and maternal complication in the group treated by vitamin-K antagonists. Anticoagulation (therapeutic or prophylactic) was associated with an increase in neonatal complications (OR $=10.0,95 \% \mathrm{CI}$ [1.5-91.4], $\mathrm{p}<0.01$ ), compared to no anticoagulation (none or antiplatelet treatment). Neonate complications occurred in $75 \%$ in the group of women under AVK during a completed pregnancy, and $81 \%$ in the group receiving LMWH, regardless of the dose, versus $25 \%$ and $38 \%$ respectively in the group of women without treatment and with antiplatelet therapy.

\subsection{Cardiac complications at last follow-up}

Median follow-up was 24 months, 95\% CI [8-31]. A total of 8 women experienced cardiac events after pregnancy. During follow-up, atrial arrhythmia occurred in 3 women at 6, 24 and 60 months after pregnancy. Among them, only one had experienced an arrhythmic event (atrial fibrillation) during pregnancy. One woman underwent pacemaker implementation for symptomatic atrioventricular block 8 months after delivery. One cerebrovascular attack occurred 18 months after pregnancy in a patient with tricuspid atresia palliated by fenestrated Fontan procedure with no prior thromboembolic episodes/AF episodes and while on antiplatelet therapy. No deaths were recorded.

\section{Discussion}

Fontan surgery and its derivatives (Kreutzer, intra-auricular cavopulmonary connection or extracardiac tube) have allowed the population with functionally single ventricle to reach adulthood in the vast majority of cases. For post-Fontan women who become pregnant, the main question is the ability of the univentricular heart to tolerate the hemodynamic transformations due to pregnancy (cardiac output increase, overload circulating volume, heart rate increase) and the risk of exacerbating the underlying dysfunctions (arrhythmias, ventricular systolic dysfunction, thromboembolic events).

Despite several anecdotal and few multicentric evidence in literature on outcomes of pregnancy in women with Fontan circulation, there is still paucity of data on the predictors of maternal and fetal outcomes especially in this subset $[9,10,14-17]$. The aim of this observational retrospective multicentric study was to determine, with a large number of pregnancies, the magnitude and frequency of these complications, with special focus on hematological complications.

Although data are missing for $8 / 37$ women, the infertility rate seems high in our population compared to women of the same age (6\%) [18]. Infertility has already been shown to be frequent in women after Fontan palliation [19] and could be explained by the combination of chronic hypoxemia before and chronic venous congestion after palliation that could result in ovarian dysfunction.

We observed a high rate of fetal loss (34\%: $7 \%$ abortion, $27 \%$ miscarriages, and one fetal death), as high as rates observed in cyanotic patients [20,21]. This high rate of miscarriages, described in other series $[9,10]$ during the first trimester, is twice that in the cardiac congenital population [22] and threefold that in the general population [23]. In our population, no predictive factor was found, neither the type of palliation, nor the treatment (including anticoagulation or anti arrhythmic), nor the NYHA functional class. However, miscarriage was more frequent under vitamin-K antagonists regardless of the dose. It is not clear, however, if ovarian dysfunction and pelvic venous hypertension have a role in this high rate of miscarriages.

Cardiovascular events during pregnancy were frequent in our population (10\%), and pre-pregnancy arrhythmias were the only predictive factors for arrhythmias during pregnancy. These arrhythmias are a frequent long-term complication of the Fontan anatomy, principally due to the enlargement of the right atrium and to the atrial surgical scars. All patients with post-Fontan operation are considered in WHO 3 condition according to the ESC task force [6], which means a significant increased risk of maternal morbidity or mortality. However risk stratification in post-Fontan operation women appears more complex and needs a larger cohort to identify specific risk factors, in addition to past medical history of arrhythmia [12,13].

The question that remains unclear is the need for anticoagulation during Fontan pregnancy. Fontan palliation is a known high-risk condition for thromboembolism, especially when the right atrium is enlarged. There is, however, no evidence for benefit to anticoagulate systematically (recommendation class IIa, level of evidence C), but anticoagulation is definitely indicated in the presence of atrial thrombus, atrial arrhythmia or history of thromboembolic events [6]. Otherwise, pregnancy and the first postpartum weeks are also in itself a known high-risk condition for thromboembolism [24]. Although we observed no fetal malformation in the group treated with AVK, risks of teratogenicity related to this treatment are well known [25]. Because our population receiving this treatment was small, and doses of AVK were not documented in our dataset, we could not draw conclusions of this result. In our series, thromboembolic events were diagnosed in $5 \%$ of pregnancies during the antepartum and postpartum periods, in women with a low risk of thromboembolic events. One of these events led to shock and required resuscitation. Because thromboembolism can lead to Fontan failure with dramatic consequences, we think prophylactic anticoagulation by LMWH in patients with low thromboembolic risk, and therapeutic anticoagulation by LMWH in women with high thromboembolic risk (i.e.: past history of arrhythmia or thromboembolic event) should be indicated, during antepartum and postpartum periods.

Among the completed pregnancies, the most common neonatal complications were prematurity (69\%) and small-for-gestational age (SGA) birth weight ( $<10$ th percentile). Among maternal congenital heart diseases, chronic cyanosis is a known risk factor for high levels of prematurity and small SGA neonates [20]. Only one of our patients was cyanotic due to a veno-venous fistula. Prematurity and SGA would then have to be explained otherwise, probably by the specificity of the Fontan circulation. Pregestational abdominal venous congestion can be a predictor of prematurity and/or SGA. Regardless of the exact cause, the very high complication rates we describe are a strong incentive for regular assessment of fetal growth during pregnancy.

Nevertheless, despite these high complication rates we describe, pregnancy in Fontan women is possible for 29 women among the 37 of our series (78\%), without maternal mortality.

\section{Limitations}

Data were retrospectively collected. Follow-up, outcome assessment, and treatment strategies were not standardized. However, information bias was likely minimized by the completeness of data reported in the study and absence of losses to follow-up. In addition, patients deemed at highest risk may have been counseled against pregnancy. For example, no patient had severe systemic ventricle dysfunction, a NYHA functional class of III or IV, or protein losing enteropathy. 


\section{Conclusion}

Our results show that pregnancy in women with Fontan palliation is possible, without maternal mortality. However, pregnancies remain at high risk for the mother with arrhythmias, and for the fetus, with a high rate of miscarriages, prematurity and SGA. Although anticoagulation therapies during pregnancy are still debated, thromboembolisms were some of the major complications we described during pregnancy or the postpartum period in women with Fontan palliation, which can threaten Fontan circulation. For these reasons, we suggest that all women with Fontan palliation should receive at least prophylactic anticoagulation during pregnancy, and the postpartum period. Preconception counseling and strict multidisciplinary monitoring by obstetrician, cardiologist and anesthetist are recommended.

\section{Conflict of interest}

None.

\section{References}

[1] F. Fontan, P. Baudet, Surgical repair of tricuspid atresia, Thorax 26 (1971) 240-248.

[2] G. Kreutzer, E. Galindez, H. Bono, C. De Palma, J.P. Laura, An operation for the correction of tricuspid atresia, J. Thorac. Cardiovasc. Surg. 66 (1975) 613-621.

[3] C. Kreutzer, J. Kreutzer, G. Kreutzer, Reflections on five decades of the Fontan Kreutzer, Front. Pediatr. 1 (2013) 45

[4] P. Khairy, S.M. Fernandes, J.E. Mayer Jr., et al., Long-term survival, modes of death, and predictors of mortality in patients with Fontan surgery, Circulation 117 (1) (2008) 85-92.

[5] Y. d'Udekem, A.J. Iyengar, J.C. Galati, et al., Redefining expectations of long-term survival after the Fontan procedure: twenty-five years of follow-up from the entire population of Australia and New Zealand, Circulation 130 (11 Suppl. 1) (2014) S32-S38.

[6] European Society of Gynecology (ESG), Association for European Paediatric Cardiology (AEPC), German Society for Gender Medicine (DGesGM), V. Regitz-Zagrosek, C. Blomstrom Lundqvist, et al., ESC Committee for Practice Guidelines. ESC Guidelines on the management of cardiovascular diseases during pregnancy: the Task Force on the Management of Cardiovascular Diseases during Pregnancy of the European Society of Cardiology (ESC), Eur. Heart J. 32 (24) (2011) 3147-3197.

[7] D.N. Rosenthal, A.H. Friedman, C.S. Kleinman, G.S. Kopf, L.E. Rosenfeld, W.E Hellenbrand, Thromboembolic complications after Fontan operations, Circulation 92 (9 Suppl.) (1995) II287-II293.
8] K. Bull, The Fontan procedure: lessons from the past, Heart 79 (1998) 213-214.

[9] M.M. Canobbio, D.D. Mair, M. van der Velde, B.J. Koos, Pregnancy outcomes after the Fontan repair, J. Am. Coll. Cardiol. 28 (1996) 763-767.

[10] W. Drenthen, P.G. Pieper, J.W. Roos-Hesselink, et al., ZAHARA investigators, Noncardiac complications during pregnancy in women with isolated congenital pulmonary valvar stenosis, Heart 92 (12) (2006) 1838-1843.

[11] L. Le Gloan, L.A. Mercier, A. Dore, et al., Pregnancy in women with Fontan physiology, Expert. Rev. Cardiovasc. Ther. 9 (12) (2011) 1547-1556.

[12] S.C. Siu, M. Sermer, J.M. Colman, et al., Cardiac Disease in Pregnancy (CARPREG) Investigators, Prospective multicenter study of pregnancy outcomes in women with heart disease, Circulation 104 (5) (2001) 515-521.

[13] W. Drenthen, E. Boersma, A. Balci, et al., ZAHARA Investigators, Predictors of pregnancy complications in women with congenital heart disease, Eur. Heart J. 31 (17) (2010) 2124-2132

[14] M. Caruana, V. Grech, J. Somerville, Pregnancy in atriopulmonary connection and total cavopulmonary connection - a comparison of two cases, Cardiol. Young 22 (2012) 600-602.

[15] S. Inoue, H. Masuyama, T. Akagi, Y. Hiramatsu, Pregnancy and delivery in patients with Fontan circulation: a report of two cases, J. Obstet. Gynaecol. Res. 39 (2001) 378-382.

[16] J.F. Nitsche, S.D. Phillips, C.H. Rose, B.C. Brost, W.J. Watson, Pregnancy and delivery in patients with Fontan circulation: a case report and review of obstetric management, Obstet. Gynecol. Surv. 64 (2009) 607-614.

[17] A. Nir, U. Elchalal, C. Hammerman, A. Rein, Twin pregnancy in a patient after the Fontan operation: report of a case, Congenit. Heart Dis. 8 (2013) E196-E198.

[18] A. Chandra, C.E. Copen, E.H. Stephen, Infertility and impaired fecundity in United States, 1982-2010: data from National Survey of Family Growth, Natl. Health Stat. Rep. 67 (2013) 1-18.

[19] M. Canobbio, D. Mair, A. Rapkin, J. Perloff, B. George, Menstrual patterns in females after the Fontan repair, Am. J. Cardiol. 66 (1990) 238-240.

[20] P. Presbitero, J. Somerville, S. Stone, E. Aruta, D. Spiegelhalter, F. Rabajoli, Pregnancy in cyanotic congenital heart disease. Outcome of mother and fetus, Circulation 89 (1994) 2673-2676.

[21] W. Drenthen, P.G. Pieper, J.W. Roos-Hesselink, et al., ZAHARA Investigators, Outcome of pregnancy in women with congenital heart disease: a literature review, J. Am. Coll. Cardiol. 49 (24) (2007) 2303-2311.

[22] P. Khairy, D. Ouyang, S. Fernandes, A. Lee-Parritz, K. Economy, M. Landzberg, Pregnancy outcomes in women with congenital heart disease, Circulation 113 (2006) 517-524.

[23] M.J. Zinaman, E.D. Clegg, C.C. Brown, J. O'Connor, S.G. Selevan, Estimates of human fertility and pregnancy loss, Fertil. Steril. 65 (1996) 503-509.

[24] N.J. Ellish, K. Saboda, J. O'Connor, P.C. Nasca, E.J. Stanek, C. Boyle, A prospective study of early pregnancy loss, Hum. Reprod. 11 (1996) 406-412.

[25] M. Cotrufo, M. De Feo, L.S. De Santo, et al., Risk of warfarin during pregnancy with mechanical valve prostheses, Obstet. Gynecol. 99 (1) (2002) 35-40. 